\title{
Copia-Like Retrotransposons in the Rice Genome: Few and Assorted
}

\author{
Carlos M. Vicient ${ }^{1}$ and Alan H. Schulman ${ }^{1,2, *}$ \\ ${ }^{1}$ Plant Genomics Laboratory, Institute of Biotechnology, University of Helsinki, Viikki Biocenter, \\ P.0. Box 56, FIN-00014 Helsinki, Finland \\ ${ }^{2}$ Crops and Biotechnology, Agrifood Research Finland, Myllytie 10, FIN-31600 Jokioinen, Finland
}

(Received: 16 October 2001; accepted: 19 December 2001)

\begin{abstract}
We performed a comprehensive analysis of Ty1-copia-like retrotransposons in about $10 \%$ of the Oryza sativa genome and identified 100 elements. These were divided into nine classes by their reverse transcriptase similarity and into 39 families according to the sequences of their long terminal repeats (LTRs). The deduced total number of copia-like elements in rice is $\sim 1000$, comprising $1.7 \%$ of the genome. Solo LTRs and truncated elements comprise an additional $2.3 \%$ of the genome. This is low compared with other plants, especially grasses, but comparable to Arabidopsis. However, the total number of families in the genome, at least 50 , is similar to that in other plants. At least some of these classes are present in the genomes of other grasses, and related elements can be found in dicotyledonous plants. The transcriptional activity of each family was estimated by searching for homologous expressed sequence tags in databases. No correlation was found between the number of homologous expressed sequence tags and family size. In comparison with the copia retrotransposons, 222 gypsy-like and 66 LINE-like retroelements, as well as five pararetrovirus-like elements, were found, corresponding to about 2220, 660, and 50, respectively, in the genome. A low rate of retrotransposon transcription and a high rate of loss of integrated copies by ectopic, intrachromosomal recombination are proposed as the reasons for the paucity of retrotransposons in the rice genome.
\end{abstract}

Keywords: Rice, Genome Evolution, Copia-like Retrotransposon, Gypsy-like Retrotransposon, LINE Element, Oryza sativa.

\section{INTRODUCTION}

Retrotransposons are mobile genetic elements that transpose through reverse transcription of an intermediate RNA. ${ }^{1}$ Retrotransposons can be divided into two main classes based on the presence or absence of long terminal direct repeats (LTRs). The LTR retrotransposons are bounded by an LTR at each end and contain an internal region coding for GAG (capsid protein), proteinase, integrase, reverse transcriptase (RT), and RNase H. Retrotransposons are structurally and functionally similar to retroviruses; both groups replicate by reverse transcription of an intermediate RNA. However, LTR retrotransposons are not

\footnotetext{
*Author to whom correspondence should be addressed.
}

infective, because of their lack of an env domain encoding an envelope glycoprotein. The LTR retrotransposons, in turn, can be divided into two main groups, the copiaand the gypsy-like elements. These differ in the order of their coding domains. Although both contain a relatively conserved $r t$ domain, sequence comparisons indicate that each group is monophyletic and transcends organismal groupings. ${ }^{2}$ The gypsy-like elements are more retroviral in their sequence as well as their coding domain organization. Non-LTR retrotransposons include LINE and SINE elements. ${ }^{3}$

The LTR retrotransposons are universal and abundant components of eukaryotic genomes in general, and of plant genomes in particular. ${ }^{4}$ Retrotransposons are present in high numbers of copies in plants, especially in those with 
large genomes, where they show considerable sequence heterogeneity. ${ }^{5-8}$ The processes of transcription and reverse transcription are error prone and lead to this heterogeneity. Furthermore, many retrotransposons within the genome are either integrated in a transcriptionally or translationally incompetent state or become fossils because of accumulation of mutations following integration. Nevertheless, the activity of retrotransposons in somatic tissues has been demonstrated, ${ }^{9}$ and a basal level of expression appears to exist for many elements. ${ }^{10}$ Of those retrotransposons quiescent in somatic tissues, many can be activated by stress, ${ }^{11,12}$ protoplast formation, ${ }^{13}$ or tissue culture. ${ }^{14,15}$ Indeed, environmental gradients involving stress have been correlated with retrotransposon-based genome differentiation in wild barley. ${ }^{16}$ Translation and formation of viruslike particles have also been directly demonstrated for plant retrotransposons. ${ }^{17}$

Unlike DNA transposons, when retrotransposons transpose, the original copy does not excise as part of the process. Retrotransposons can thereby greatly increase their copy numbers and, consequently, their genome size. ${ }^{18}$ Whereas retrotransposons account for $50-80 \%$ of the large maize genome (Zea mays L, $\left.1 C=2.6 \times 10^{9} \mathrm{bp}\right),{ }^{19}$ the small Arabidopsis thaliana L. genome $\left(1 C=1.3 \times 10^{8}\right)$ contains about $5.5 \%$ retrotransposons in the finished sequence (T. Bureau, personal communication). The control or inefficiency of retrotransposition, however, is not the only possible explanation for the paucity of retrotransposons in small genomes. Mechanisms such as recombination between LTRs can eliminate retrotransposon sequences from the genome, generating solo LTRs and other types of deletion products. ${ }^{20,21}$

Cultivated rice (Oryza sativa L.) has one of the smallest genomes $\left(1 C=4.3 \times 10^{8} \mathrm{bp}\right)^{22}$ in the genus Oryza, which is composed of more than 20 species that vary 3.5 -fold in nuclear DNA content, from $3.43 \times 10^{8}$ to $12.01 \times 10^{8} \mathrm{bp}$ among the diploids. ${ }^{19}$ Three times that of $A$. thaliana, the rice genome is also among the smallest of the grasses as a whole, which range from $2.0 \times 10^{8}$ bp in Oropetium thomaeum to $1.3 \times 10^{10}$ bp in Lygeum spartum. ${ }^{19}$ In most grasses, genes comprise less than $20 \%$ of the genome, ${ }^{23}$ the rest being composed mainly of repetitive DNA. Gene number is thought to be relatively constant within groups of related species, and, consistent with this, most of the 3.6-fold difference in genome size between maize and sorghum was shown to be due to the accumulation of retrotransposons. ${ }^{24}$

Such comparisons lead to the expectation that the rice genome should contain less repetitive DNA generally and fewer LTR retrotransposons in particular. Early solution hybridization experiments indicated that only half of the rice genome is composed of repetitive sequences. ${ }^{25}$ Based on the screening of genomic libraries with a conserved retrotransposon probe ( ${ }_{\text {met }}^{i}$ tRNA primer), Hirochika and co-workers ${ }^{26}$ estimated that the rice genome contains about
1000 retrotransposons in total, divided into 32 families. By hybridizing a rice BAC library with $r t$ probes representative of different $r t$ families, Wang and co-workers, ${ }^{27}$ however, estimated that the genome contains only about 100 copia-like elements. A detailed survey of $910 \mathrm{~kb}$ of rice genome $(0.2 \%)$ revealed two copia-like and 10 gypsy-like retrotransposons, ${ }^{28}$ giving a total estimation of 1000 copia and 5000 gypsy elements in the genome, representing $2.6 \%$ and $7.3 \%$ of the total genome, respectively. They also detected five non-LTR retrotransposons, giving a total estimate of 2500 elements in the genome.

These estimates span a 100-fold range, leaving the issue of retrotransposon copy number in rice unresolved. Furthermore, although various retrotransposon families have been reported for rice, ${ }^{26,29-34}$ the total number of families present in the genome remains unclear. The current availability of an appreciable part of the sequence of the rice genome provides an excellent and timely opportunity to analyze the evolutionary dynamics of rice retrotransposons. In this study, we examine the sequence abundance and diversity of the rice copia-like, LTR-containing retrotransposons.

\section{EXPERIMENTAL METHODS}

\subsection{Isolation of Rice Retrotransposon Sequences}

We used a collection of amino acid sequences corresponding to reverse transcriptases of copia-like elements. ${ }^{5,6,8,35-37}$ These sequences were used in TBLASTN searches, ${ }^{38}$ where translated nucleotide databases are queried by a polypeptide string, through the on-line facilities of the National Center for Biotechnology Information (NCBI) (http://www.ncbi. nlm.nhm.gov/). The plant division of the Genbank database (Release 125.0, 15.08.2001), specifically all O. sativa PAC, YAC, and BAC accessions, which are the annotated long contiguous chromosomal regions, was queried. New reverse transcriptase (RT) sequences were extracted from the database with this approach. These sequences were again used as queries in additional database searches, carrying the process forward iteratively until no new RTs were obtained. The limits of the RT domain were defined according to the method of Xiong and Eickbush, ${ }^{2}$ but only the central, most conserved "core" of this domain was used in multiple alignments, as has been used in previous analyses based on polymerase chain reaction (PCR) amplification. ${ }^{5,6}$ We did not exclude $r t$ sequences with frameshifts and stop codons from our analyses. LTR sequence searches were conducted on-line with the BLAST2 program (http://www.ncbi.nlm.nih.gov/ gorf/bl2.html).

For gypsy-like retroelements, the same method was followed as for the copia-like ones, with RIRE2 (accession AB030283.1, nt 3650-4180) used as the initial query sequence to seed the iterative search procedure. For LINElike retrotransposons, the searches were seeded with the 
RT-encoding domains of Li2 (Y13371) and Li4 (Y13370) elements, isolated from rice. ${ }^{39}$ Integrated pararetroviruses were discovered as a by-product of the search for gypsy-like sequences and were distinguished from them by sequence comparisons.

\subsection{Sequence Comparisons and Phylogenetic Trees}

CLUSTALW of the EMBL European Bioinformatics Institute web page (http://www.ebi.ac.uk/clustalw/) ${ }^{40}$ was used to generate multiple sequence alignments of the predicted RT polypeptides, which were edited manually to correct errors. The TREECON $1.3 \mathrm{~b}$ program ${ }^{41}$ was used to generate phylogenetic trees of the predicted polypeptides based on the neighbor-joining method. ${ }^{42}$ Branch reliability in the phylogenetic predictions was tested by bootstrap analysis, based on 1000 repetitions. Homology searches of expressed sequence tags (ESTs) were made as described before. ${ }^{10}$

\section{RESULTS}

\subsection{Identification and Frequency of Copia-Like Retrotransposons in the Rice Genome}

The diversity of the copia-like retrotransposons in the genome of $O$. sativa was examined by a systematic search of all rice sequences available in Genbank, representing about $10 \%$ of the rice genome. The RT domains were used for query sequences. Because the core domain of RT is highly conserved and sequences of diverse origin have been aligned for phylogenetic analyses, the primary structure is a reliable tool for identifying retrotransposons. The predicted RTs identified in Genbank accessions were iteratively applied in further queries until no new copia-like coding regions were found. A total of 182 nonredundant, complete $r t$ regions were identified. Of these, 81 corresponded to PCR products, one was a cDNA, and 100 were genomic sequences.

Assuming that the $10 \%$ of the genome surveyed is representative of the whole, the total number of copia-like retrotransposons in rice would be about 1000, comprising about $1.7 \%$ of the total genome. This is likely to be an underestimation of the total number of copia-like retroelements, however, because our count includes only elements with complete $r t$ domains. In some species such as barley, solo LTRs can contribute as much DNA to the genome as the intact elements themselves. ${ }^{20}$ In an attempt to estimate how many of these "RT-defective" elements are present in the genome, we searched databases with the BLAST program and the sequences of each rice retrotransposon as queries. By this method, the 1210 copia-like solo LTRs and 380 truncated retroelements represent about $0.6 \%$ of the genome, bringing the copia-like elements to $2.3 \%$ of the nuclear DNA. This is very much more than some previous estimates ${ }^{26,27}$ but more similar to other, sequencebased estimates. ${ }^{28}$ In our view and that of others, ${ }^{28}$ the earlier underestimations were due to the use of nucleic acid hybridizations that probably did not detect some of the families present in the genome. Discrepancies in computerbased estimations could be explained by differences in the source and quantity of DNA sequences used.

\subsection{Families and Phylogeny of Copia-Like Retrotransposons in the Rice Genome}

We analyzed the phylogenetic relationships between the 100 rice genomic $r t$ domains by constructing a neighborjoining tree, displayed in Figure 1, on the predicted polypeptides, using the deduced amino acid sequences. Sequences sharing more than $20 \%$ amino acid similarity were grouped into classes. The sequences grouped into nine major classes, each containing from one to 31 elements. Elements in the same class in general also share similar $(-)$-strand (PBS) and (+)-strand (polypurine tract, PPT) primer binding sites. The elements in most of these classes could be subdivided into different families. We used LTR sequence similarity as the criterion to define the families for several reasons. The LTR is the most rapidly evolving region of retrotransposons ${ }^{43,44}$ and therefore gives good phylogenetic resolution. The LTR critically not only determines transcription patterns, which can differ between retrotransposon families, ${ }^{11}$ but also provides the recognition sites for integration. By our criterion, two elements belong to the same family if their LTR sequences share more than $90 \%$ of DNA sequence homology in at least $100 \mathrm{bp}$.

We examined the flanking sequences of each of the $r t$ domains to identify potential LTRs. The LTRs were delimited by the presence of sequences similar to the conserved PBS, expected to be next to the $5^{\prime}$ LTR; by the PPT, expected to be adjacent to the $3^{\prime} \mathrm{LTR}^{1}$; and by the direct repeat outside of the LTRs in the host DNA, which are generated by integration. In some cases either it was not possible to identify some of these features or one of the LTRs was truncated, missing, or outside the insert of the clone. Despite these limitations, LTRs were identified for most of the coding domains by comparisons with other members of the same family or related sequences.

We also studied the RIRE1 element in a bit more detail. RIRE1 is a copia-like retrotransposon present in high copy number in wild rice Oryza australiensis [31]. For the RIRE1 element (family 6C), we found three elements containing RT and LTRs, 25 solo LTRs, and one element with LTRs but a deletion in the RT domain. That gave us a total of 33 LTRs and four internal regions, and an estimation of 330 RIRE1 LTRs in the genome, not very different from the previous estimation of 180 RIRE1 LTRs. ${ }^{29}$

In all of the cases in which two elements had the same type of LTR, they were located in the same branch of the 


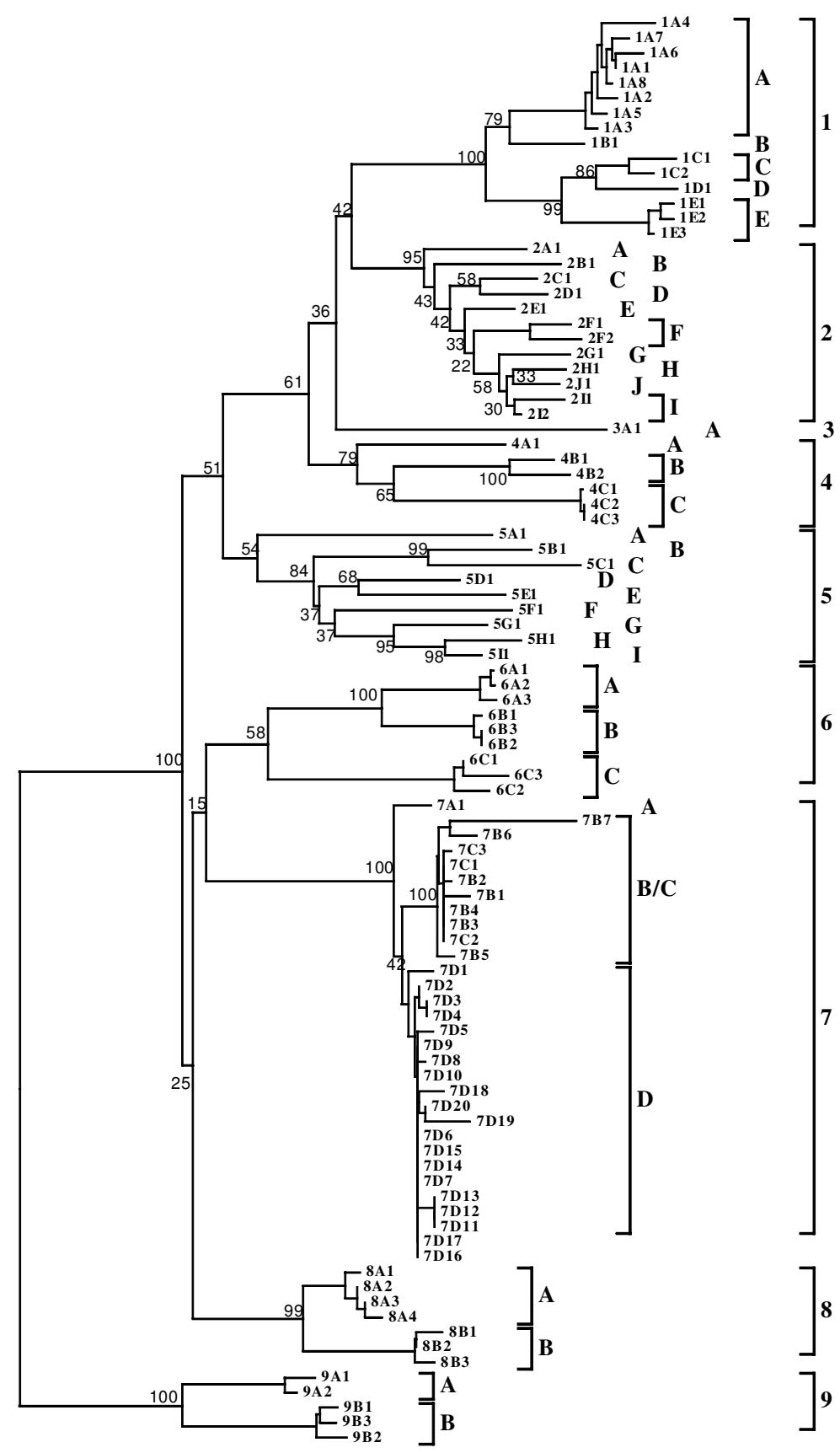

Figure 1. Neighbor-joining tree of the reverse transcriptase amino acid sequences of copia-like retrotransposons in the rice genome. Genbank accession numbers and the position in the sequence are indicated. To the right of the various lineages, the classes of elements are indicated by numbers, and the families are indicated by letters. Bootstraps were performed with 1000 repetitions; bootstrap values (as a percentage) are shown next to branches dividing classes and families of sequences.

RT-based tree. We therefore believe the RT-based classification to be robust enough to be used to divide the copia-like retrotransposons into classes, and we used LTRs only to define within-class families. The only exception were families 7B and 7C, which have highly similar RTs but different LTR sequences. A total of 39 families were identified, as shown in Figure 1. In general, as described in Table 1, families in the same class have similar total sizes, similar LTR lengths, and similar PBS and PPT sequences. For example, all four families in Class 1 have LTRs longer than $1200 \mathrm{bp}$ and total sizes around $9 \mathrm{~kb}$, whereas the nine families in Class 5 have LTRs between 230 and 474 bp and internal domain lengths between 4.1 and $5.1 \mathrm{~kb}$. The most abundant families are 7C and 7B, with 22 and 11 elements, respectively. For several families, we found only one element.

Some copia-like retrotransposons have previously been characterized in rice, and several PCR fragments corresponding to copia-like $r t$ domains have been sequenced 
Table 1. Occurrence of copia-Like retrotransposons in Oryza sativa genomic sequences.

\begin{tabular}{|c|c|c|c|c|c|c|c|c|}
\hline Element & Size & LTR & Accession & Position & $5^{\prime}$ flank & 3' flank & PBS & $\begin{array}{l}\text { PPT } \\
\text { PPT }\end{array}$ \\
\hline \multicolumn{9}{|c|}{ Class 1} \\
\hline $1 \mathrm{~A} 1$ & 6850 & $1199 / 1221$ & AC027661.1 & $30253-39522$ & TСТСATECAT & TECAT $_{A G T T T}$ & AGTGGTATCACAGCCT & TCATCAAGGGGGAGAT \\
\hline $1 \mathrm{~A} 2$ & 6853 & $1190 / 1219$ & AC068654.2 & $143638-152889$ & TAGCCTIAAT & TTAAСтTCTA & AGTGGTATCACAGTCT & TCATCAAGGGGAGAT \\
\hline $1 \mathrm{~A} 3$ & 8894 & $231 / 1197$ & $\mathrm{AC} 074283.3$ & $68152-78473$ & CGATATCCAG & ССТСТСАТGT & AGTGGTATTACAGCCA & TCATCAAGGGGAGAT \\
\hline $1 \mathrm{~A} 4$ & 6778 & $1174 / 1167$ & AC078839.4 & $102270-110504$ & GCGCGCGGAA & CGGARTAGTG & AGTGGTATCACAGCCT & TCATTGAGGGGAGAT \\
\hline 1A 5 & 6820 & $1213 / 1212$ & AP002069.1 & $68612-77856$ & ATATGTACAT & CAGATATCCA & AGTGGTATCACAGCTT & TCATTAAGGGGAGAT \\
\hline $1 \mathrm{~A} 6$ & 6801 & $1214 / 1156$ & AP002971.2 & $14644-22261$ & СтСтСATTE & ATTTGCATAT & AGTGGTATCACAGCCT & TCATCAAGGGGAGAT \\
\hline 1A 7 & 6781 & $1215 / 1220$ & AC021891.6 & $65191-74406$ & AAGTCGITTA & АTTTAАСТTTT & AGTGGTATCACAGCCT & TCATCAAGGGGAGAT \\
\hline $1 \mathrm{~A} 8$ & 6596 & $812 / 1145$ & AP003073.1 & $126193-132708$ & CGGAGGTTCC & ATTGGGCGTT & AGTGGTATCACAGCCT & TCATCAAGGGGAGAT \\
\hline 1B1 & 5672 & $1618 / 1630$ & $\mathrm{AP} 002538.2$ & $44996-53915$ & ACCTTAE' & AE'tuCTCCT & ATTGGTATCACAGCCC & CCATCAAGGGGAGAT \\
\hline $1 \mathrm{C} 1$ & 8074 & $1424 / 1429$ & AC084319.5 & $70748-85600$ & CACAAAAALIA & AAALIACAGTA & ATTGGTATCGAAGCGA & CCAAAAGAGGGGAGAT \\
\hline $1 \mathrm{C} 2$ & 8208 & $1432 / 134$ & AC091238.2 & $71552-71326$ & TTAGTCTATC & $\mathrm{cccccccccC}$ & n.a. & n.a. \\
\hline $1 \mathrm{D} 1$ & 6209 & $1794 / 1677$ & AP000969.1 & $23381-33059$ & ССТТСАACTC & AАСТCTTATA & ATTGGTATGAGAGCCT & CTAAAAAGGGGGAGAT \\
\hline $1 \mathrm{E} 1$ & 8831 & $1580 / 1585$ & $\mathrm{AC} 069324.3$ & $132725-144720$ & TCTCTAGCCT & TTTCATCGGG & AGTGGTATCAGAGCTT & CCAAAAAGGGGAGAT \\
\hline $1 \mathrm{E} 2$ & 8645 & $1198 / 1396$ & AP003205.2 & $49512-49734$ & GGGGAGTCAT & GICAT $C T C A G$ & n.a. & AAAAAAAGGGGAGAT \\
\hline $1 \mathrm{E} 3$ & 8004 & $1669 /$ n.a. & AP003199.3 & $29060-36796$ & AAAACGCTGG & ТTСАтстTтT & AGTGGTATCACAGCTT & n.a. \\
\hline \multicolumn{9}{|c|}{ Class 2} \\
\hline $2 \mathrm{~A} 1$ & 5069 & $226 / 226$ & AC087181.8 & $83222-88742$ & CGTGCGATCA & GGATGGAACC & TGGTATCAGACEGTTG & ATCAGAATCCACTCCC \\
\hline $2 \mathrm{~B} 1$ & 4398 & $206 / 172$ & $\overline{A C 068654.2}$ & $23236-28011$ & GGGAGGGCAC & GGCACCACCG & TGGTATCAGGCCCTAT & ATGATTGAGGGGGGC \\
\hline $2 \mathrm{C} 1$ & 4424 & $226 / 226$ & $\mathrm{AP003289.3}$ & $122532-127407$ & GAGTCAACAA & AACAAGACAA & TGGTATCAGACCTTTT & TATGATTGAGGAGGG \\
\hline $2 \mathrm{D} 1$ & 4356 & $209 / 209$ & $\mathrm{AP002843.2}$ & $139482-144255$ & GTGTTATCCT & ATCCTCCTTC & TGGTATCAGACCCATA & TGTGATTGAGGAGGA \\
\hline 20 & 4426 & $177 / 177$ & $\mathrm{AC} 087544.2$ & $66943-71722$ & ATGATEICEG & EICEGCATGG & TGGTATCAGACCCTC & TGTGATTGAGGIGGAG \\
\hline $2 \mathrm{~F} 1$ & 4347 & $180 / 183$ & $\overline{\mathrm{AP} 002093.1}$ & $28354-33063$ & TAGTITGCCA & TGECA & TGGTATCAGAGCCTCC & TAAGATTGAGGGGAG \\
\hline $2 \mathrm{~F} 2$ & 4387 & $191 / 191$ & AC074283.3 & $19794-24562$ & AGGTGGGTTC & GGTTCATACA & TGGTATCAGACCCTTA & TGCAATTGAGGGGAG \\
\hline $2 \mathrm{G} 1$ & 4379 & $218 / 218$ & $\overline{\mathrm{AC} 0091123 .}$ & $7841-8181$ & AACGCCGTCC & CGTCCGATTT & TGGTATCAGACAAAAA & TGAGATTGAGGGGAG \\
\hline $2 \mathrm{H} 1$ & 3579 & $73 / \cdots$ & $\mathrm{AP} 003046.2$ & $100588-104239$ & TATGTATGTA & TGTAGCGCCC & TGGTATCAGACCTTC & AGAGTAGGAATGAATG \\
\hline 2 I1 & 4410 & $221 / 221$ & $\overline{A C 016781.6}$ & $26002-30853$ & GTGACCTCGG & CTCGGCGTTG & TGGTATCAGACCACCC & TAAGATTGAGGGGAG \\
\hline 2 I 2 & 5437 & $220 / 220$ & AC016781.6 & $72407-78283$ & СТGСАCACTG & САСтGGTCCC & TGGTATCAGACCCTGT & TAAGATTGAGGGGAG \\
\hline $2 \mathrm{~J} 1$ & 4330 & $184 / 205$ & AC069300.7 & $73013-77731$ & GTCAAGHCCC & GICCCTCCTG & TGGTATCAGACCCTCC & TCAGATTGAGGGGAG \\
\hline \multicolumn{9}{|c|}{ Class 3} \\
\hline 3A1 & 3928 & $138 / 138$ & $\mathrm{AC} 087545.2$ & $80982-85185$ & ТТСТТСТCT & СтсстTтстT & TGGTATCAGACAATG & TGAGTTTGAAGGGGG \\
\hline \multicolumn{9}{|c|}{ Class 4} \\
\hline $4 \mathrm{~A} 1$ & 3116 & $135 / 135$ & $\mathrm{AP} 002872.2$ & $90440-90179$ & CAGCTEAACA & GAACATATTT & n.a. & TTAGACTGAGGGGAG \\
\hline 4B1 & 4672 & $199 / 235$ & AF161269.1 & $108089-113194$ & TAAACCAII'I & CAIIITGACTT & TGGTATCAGACCTAG & TGCGACTGCGGCGGG \\
\hline $4 \mathrm{~B} 2$ & 6042 & $201 / 203$ & $\mathrm{AC} 027661.2$ & $138699-145144$ & GATCAACTAT & ACTATATATT & TGGTATCAGAGTTCT & TACGACTGCGGCGGG \\
\hline $4 \mathrm{C} 1$ & 2105 & $221 / 221$ & AC079037.3 & $33655-36201$ & GAAGGCGCCC & CGCCCAGTGC & TGGTATCAGTCACCCT & TGCGACTGCGGGGGG \\
\hline $4 \mathrm{C} 2$ & 4276 & $239 / 221$ & AC0 73166.7 & $104290-109025$ & TGTTTCTTET & СтTCTATTGT & TGGTATCAGTCACCCT & TGCGACTGCGGGGGG \\
\hline $4 \mathrm{C} 3$ & 4277 & $221 / 221$ & AC025098.4 & $12386-17104$ & тстт ATATG & ATATGTTTAT & TGGTATCAGTCACCCT & TGCGACTGCGGGGGGG \\
\hline \multicolumn{9}{|c|}{ Class 5} \\
\hline $5 \mathrm{~A} 1$ & 4200 & $361 / 361$ & $\mathrm{AC037197.2}$ & $66391-71188$ & CCGTCGACET & GACEICGCCG & ACTGGTATCACAGCGC & AAATTAAGGGGAGTT \\
\hline $5 \mathrm{~B} 1$ & 4334 & $472 / 476$ & $\overline{A L 512543.1}$ & $49668-54949$ & CAAAGGCEAC & GCCACACCTA & $\overline{\text { AGTGGTATCAAGCAC }}$ & TAATTAAGGGGAGAT \\
\hline $5 \mathrm{C} 1$ & 5138 & $477 / 477$ & AC021891.6 & $56044-62135$ & TAGTCTACAG & TACAGCCCGT & AGTGGTATCACAGCTT & GAATTAAGGGGGAGTT \\
\hline $5 \mathrm{D} 1$ & 4325 & $439 / 439$ & AP001366.1 & $57570-62772$ & CGGTGCAGGT & CAGGTGGTGT & ATTGGTATCAAGCAG & AGATTAGGGGGAAAT \\
\hline $5 \mathrm{E} 1$ & 4185 & $415 / 415$ & $\mathrm{AC} 080019.7$ & $55421-59541$ & CCTGCAAGGC & AAAGGCGTCAT & TTTGGTATCAGAGCTT & AGATTAGGGGGIGAAT \\
\hline
\end{tabular}


Table 1. Continued

\begin{tabular}{|c|c|c|c|c|c|c|c|c|}
\hline $5 \mathrm{~F} 1$ & 4336 & $436 / 436$ & AP003435.1 & $40436-45643$ & ССТССAEGTC & AEGTCCGTAT & AGTGGTATCACAGCCT & GCTTAGGGGGIGATT \\
\hline 5G1 & 4339 & $250 / 268$ & AL442110.1 & $38597-43453$ & CACAACACCC & CACCCATAGG & ATTGGTATCACAGCTC & $\begin{array}{l}\text { GGATTAGGGGCAGAAT } \\
\end{array}$ \\
\hline $5 \mathrm{G} 2$ & 4414 & $268 / 268$ & AL442110.1 & $95120-100070$ & GGCTCATATT & ATATITCTCA & ATTGGTATCACAGCTC & GGATTAGGGGCAGAAT \\
\hline $5 \mathrm{H} 1$ & 4937 & $229 / 286$ & AC079736.1 & $85122-90573$ & TAGCTTATAA & TATAAAAGTA & ATTGGTATCATAGCAA & AGACTTAGAAGGAGAA \\
\hline 5 I1 & 4965 & $350 / 350$ & $\mathrm{AB} 026295.2$ & $160208-165872$ & AGGTOETTAC & GTTACACTTT & TCTGGTATCACAGCAA & AGACTTAGGGGAGAA \\
\hline 5 I 2 & 3252 & n.a./131 & AC074354.8 & $130053-133141$ & n.a. & CTCGAGTCGA & n.a. & n.a. \\
\hline 5 I 3 & 4978 & $362 / 362$ & AP002836.1 & $135483-141185$ & AGGT CCACAT & CACATGACTC & TCTGGTATCACAGCCA & AGACTTAGGGGAGAA \\
\hline $5 \mathrm{I} 4$ & 4974 & $363 / 363$ & AC0 84763.4 & $95584-101284$ & ACGTTGCAAT & GCAATTACAC & TCTGGTATCACAGCCA & AGACTTAGGGGGAGAA \\
\hline 5 I 5 & 4947 & $396 / 395$ & AP000836.1 & $56024-61762$ & TTATCAAATE & AAATETACTA & TCTGGTATTACAGCCA & AGACTTAGGGGGAGAA \\
\hline \multicolumn{9}{|c|}{ Class 6} \\
\hline $6 \mathrm{~A} 1$ & 6087 & $402 / 402$ & AP003338.2 & $90661-97551$ & AAGAATCCAC & TCCAC $C A G A T$ & ATGGTATCAGAGCACC & CGATCAAGGGGAGAA \\
\hline $6 \mathrm{~A} 2$ & 6105 & $401 / 402$ & AP002845.1 & $42644-49551$ & СTGTATECTT & TECTIAACAT & ATGGTATCAGAGCACC & CGATCAAGGGGGAGAA \\
\hline $6 \mathrm{~A} 3$ & 6117 & $405 / 403$ & $\operatorname{AC} 091238.2$ & $117675-124599$ & GGGCGCGCAC & CGCACCAGAT & ATGGTATCAGAGCACC & CGATCAAGGGGGAGAA \\
\hline 6B1 & 5417 & $483 / 482$ & AF161269.1 & $87038-87390$ & AAGTTACTCA & ALTCRTGGT & ATGGTATCAGRGCAAC & AGATCAAGGGGAGAA \\
\hline $6 \mathrm{~B} 2$ & 5471 & $492 / 492$ & AP003607.3 & $7372-13826$ & СТСАТСCCAC & CСCAC $\triangle T C C T$ & TTGGTATCAGAGCAAC & AGATCAAGGGGGAGAA \\
\hline $6 \mathrm{~B} 3$ & 5453 & $485 / 485$ & AP0 003631.2 & $16304-22726$ & AAGAAEEGEA & GEGEA $A$ TGAT & ATGGTATCAGAGCAAC & AGATCAAGGGGGAGAA \\
\hline $6 \mathrm{C} 1$ & 6102 & $1399 / 1341$ & AP003257.3 & $2488-11329$ & GGGGGETAAT & GTAATAACCA & n.a. & GGAGAC \\
\hline $6 \mathrm{C} 2$ & 5212 & $1017 / 1079$ & $\mathrm{AC} 084831.6$ & $66126-73433$ & AGGATGETEG & TETCETAGGC & GGTGCTAGCACATCTG & AGTGCAAGTGGGAGAC \\
\hline $6 \mathrm{C} 3$ & 6209 & $216 / 1368$ & AP003203.2 & $3488-3911$ & ACGAGCTCAC & TGCTATCGTA & GTGGTATCAGAGCCAC & AGTGCAAGTGGGAGAC \\
\hline \multicolumn{9}{|c|}{ Class 7} \\
\hline 7A1 & 4458 & $858 / 858$ & AP003537.2 & $115701-121875$ & GGAGGEATAT & GATATAACCA & TTCCAGAAACCITTAG & TGAAA \\
\hline 7B1 & 4468 & $986 / 983$ & $\mathrm{AP} 003213.3$ & $139586-146023$ & TGGTGGGGAT & GTGTTTATTA & ATCCAAAAAATCIGATA & TGGGAT \\
\hline $7 \mathrm{~B} 2$ & 4463 & $961 / 990$ & AP002486.1 & $83267-88211$ & ATGTGIAATC & 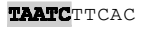 & ATCCAAAAACCIGATA & GAAATTTGGTGGGAT \\
\hline 7B3 & 4470 & $1503 / 1001$ & AP002843.2 & $43107-52941$ & TGGGAGTTEG & GTTEGTCGTC & ATCCAAAAACCTGATA & GAAATTTTGGTGGGAT \\
\hline 7B 4 & 4468 & $978 / 963$ & AC025296.1 & $158281-164690$ & TTAGCT CATA & ATATACTGGT & ATCCAAAAACCTGATA & GAAATTTGGTGGGAT \\
\hline $7 \mathrm{~B} 5$ & 4468 & $976 / 998$ & AP003256.1 & $153934-160376$ & CCGCCGCCAT & GTTGTCTATA & ATCCAAAAACCIGATA & GAAATTTTGGTGGGAT \\
\hline $7 \mathrm{~B} 6$ & 4626 & $395 / 1066$ & AP003257.3 & $76175-83456$ & TGGAGTAAAC & TAAACATGAT & n.a. & GAAATT T GGT AGGGAT \\
\hline 7B 7 & 2330 & $118 / 119$ & AC051624.6 & $25238-29317$ & TTGCTGCTTC & AGCTATTAGA & n.a. & n.a. \\
\hline $7 \mathrm{C1}$ & 4469 & $965 / 965$ & $\mathrm{AC} 023240.8$ & $100409-106808$ & GGGGAAGTCC & AGTCC $G G C A A$ & ATCCAAAAACCT GATA & AAAATTTGGTGGGAT \\
\hline $7 \mathrm{C} 2$ & 4461 & $965 / 965$ & AP000969.1 & $113971-120362$ & ATGT CCATAT & CATATGGCTC & ATCCAAAAACCTGATA & AAAATTTGGTGGGGAT \\
\hline $7 \mathrm{C} 3$ & 4471 & $1148 / 1003$ & $\operatorname{AC} 083945.3$ & $37356-43978$ & CCGGACATAC & CATACAGCAT & GTCCAAAAACTTGATA & GAAATT T TGGT RGGAT \\
\hline 7D1 & 4504 & $968 / 979$ & $\mathrm{AP} 002883.1$ & $52500-58741$ & CGCTCIANRTI & TARRITTAATT & ATCCAAAAACCITATA & AGATTTGGTGGGGAT \\
\hline $7 \mathrm{D} 2$ & 4501 & $975 / 985$ & AP 003144.1 & $85756-92221$ & ATGAACTEGC & CTEGC $A$ GTGA & ATCCAAAAACCITATT & AGATTTGGTGGGGAT \\
\hline 7D 3 & 4501 & $980 / 995$ & AP003021.2 & $89259-95734$ & AGGCAETEES & GTEEGCATAC & ATCCAAAAACCITATT & AGATTTGGTGGGGAT \\
\hline 7D 4 & 4501 & $970 / 968$ & AP 003202.2 & $44254-50692$ & TGGCTCTEAC & CTEA GATCGC & ATCCAAAAACCITATT & AGATTTGGTGGGGAT \\
\hline 7D 5 & 4500 & $968 / 978$ & AC073405.2 & $72924-79369$ & TGAAATATET & TACETAAAAC & ATCCAAAAACCITATT & AGATTTGGTGGGGAT \\
\hline 7D 6 & 4501 & $913 / 958$ & AP002872.2 & $58702-65073$ & AGGTAGAAGA & GAAGA $A A C C A$ & ATCCAAAAACCITATT & AGATTTGGTGGGGAT \\
\hline 7D 7 & 4501 & $900 / 926$ & AP003118.2 & $23292-29618$ & GAGAGEAACT & GAACTAATAT & ATCCAAAAACCITATT & AGATTTGGTGGGGAT \\
\hline 7D 8 & 4501 & $869 / 973$ & AP000836.1 & $10914-17390$ & AGGCTECATT & GCATITAGTT & ATCCAAAAACCITATT & AGATTTGGTGGGGAT \\
\hline 7D 9 & 4501 & $963 / 978$ & AP003143.2 & $124008-130449$ & ACTAATATEC & TATECTTTAT & ATCCAAAAACCITATT & AGATTTGGTGGGGAT \\
\hline 7D10 & 4501 & $938 / 977$ & AP 003504.1 & $61735-68150$ & CAGGTETEAE & GTEAECATCT & ATCCAAAAACCITATT & AGATT'TGGTGGGGATT \\
\hline 7D11 & 4501 & $941 / 980$ & $\operatorname{AC} 087546.1$ & $95510-101931$ & GСCT ССETTT & CETITTTCTG & ATCCAAAAACCITATT & AGATTTGGTGGGGAT \\
\hline 7D12 & 4499 & $780 / 964$ & AC087546.1 & $28857-33217$ & GGTGGGGGAT & GTССTCCCCC & ATCCAAAAACCITATT & AGATT'TGGAGGGGAT \\
\hline 7D13 & 4498 & $861 / 969$ & AC087546.1 & $160052-162204$ & TAGCAACATC & TCATCTACCA & ATCCAAAAATCLTATT & AGATTTGGTGGGGAT \\
\hline 7D14 & 4501 & $971 / 983$ & AP002914.2 & $136611-143065$ & CTGAAGAAAG & GAAAGAGGAA & ATCCAAAAACCITATC & AGATT'TGGTGGGGAT \\
\hline 7D15 & 4501 & $953 / 968$ & AC087192.1 & $88449-94870$ & CACAAEAAAT & GAAAAT TGCCC & ATCCAAAAACCITATT & AGATTTGGTGGGGAT \\
\hline
\end{tabular}


Table 1. Continued

\begin{tabular}{|c|c|c|c|c|c|c|c|c|}
\hline 7D16 & 3866 & $958 / 962$ & AP003104.2 & $23131-28916$ & СААТАСТTTA & СтTIAACGAA & ATCCAAAAAACCITATT & AGATTTGGTGGGGAT \\
\hline 7D17 & 4477 & $915 / 916$ & AC0 87797.5 & $13217-19524$ & CTAATEACTA & GACTATTTAT & ATCCAAAAAACCITATT & AGATTTGGTGGGGAT \\
\hline 7D18 & 2909 & $710 / 950$ & $\operatorname{AF} 172282.1$ & $129515-134083$ & TACATATATA & ATATATTGCT & n.a. & AGATTTGGTGGGGAT \\
\hline 7D19 & 1923 & n.a./953 & AL512543.1 & $81114-1119$ & n.a. & ACTCC $\mathrm{CCAGG}$ & n.a. & AAATTTGGTGGGGAT \\
\hline $7 \mathrm{D} 20$ & 4501 & $957 / 978$ & AC087852.9 & $50420-56855$ & ATGCTTIAAC & TTAACCTGTA $_{T}$ & ATCCAAAAACCITATT & AGATTTGGTGGGGAT \\
\hline \multicolumn{9}{|c|}{ Class 8} \\
\hline $8 \mathrm{~A} 1$ & 4082 & $319 / 319$ & AC069324.3 & $8813-10028$ & GTGCIGEGAG & GECAGTGGAG & AGTGGTATCACAGCTT & GTCTCCAGGTGGATT \\
\hline $8 \mathrm{~A} 2$ & 4108 & $249 / 46$ & AC079853.2 & $126402-130804$ & CGGTCTCCEC & $T \mathbf{T C C C G T A G}$ & AGTGGTATCACAGCTT & GTCTCAAGGTGGAGTT \\
\hline $8 \mathrm{~A} 3$ & 4109 & $316 / 329$ & AP003263.2 & $179142-183895$ & GGCGGCTACC & СТАCC TCCAC & AGTGGTATTACAGCTT & GTCTCAAGGTGGAGTT \\
\hline $8 \mathrm{~A} 4$ & 4108 & $259 / 297$ & AP003683.2 & $49289-53952$ & GGGCTTAEAG & TGEAGGAGGC & AGTGGTATCACAGCTT & GTCTCAAGGTGAGTT \\
\hline 8B1 & 4546 & $262 / 262$ & $\mathrm{AP} 002747.2$ & $39882-44951$ & GGGTGECTTC & GСTTCTTCCT & AGTGGTATCACAGCAT & GTCTCAAGGTGGAGAT \\
\hline $8 \mathrm{~B} 2$ & 4567 & $247 / 259$ & AP003311.2 & $168526-173598$ & ACAGTGGCAG & GGCAGATAGA & AGTGGTATCATAGCAT & GTCTCAAGGTGAAGAT \\
\hline $8 \mathrm{~B} 3$ & 4488 & $263 / 263$ & $\operatorname{AC} 090056.2$ & $33496-38509$ & CTGGACCACG & CCACGAACGC & AGTGGTATCACAGCAT & GTCTCAAGGTAGAGAT \\
\hline \multicolumn{9}{|c|}{ Class 9} \\
\hline 9A1 & 7036 & $296 / 295$ & AP002871.2 & $94011-101637$ & GTACTATGCA & TATACTACTT & CGTTATTAGCACGAGG & TTCGCCCAAGGGGAG \\
\hline $9 \mathrm{~A} 2$ & 6970 & $295 / 295$ & AC084404.8 & $105862-113421$ & GATT CCTECT & СTECT $G T C C G$ & CGTTATCAGCACGAGG & TTCACCCAAGGGGGAG \\
\hline $9 \mathrm{~B} 1$ & 7191 & $275 / 257$ & $\operatorname{AF} 172282.1$ & $9612-17334$ & TCTTCETT'TA & GTTIAAGTCT & CGTTATCAGCACTTTC & TTCGATCAAGGGGAG \\
\hline $9 \mathrm{~B} 2$ & 5087 & n.a. $/ 252$ & AP002866.1 & $161415-166753$ & n.a. & TTTTT TACAG & n.a. & TTCGATCAAGGGGGAG \\
\hline $9 \mathrm{~B} 3$ & 3477 & n.a./261 & AP002969.1 & $240-3977$ & n.a. & EATCA $G A C C A$ & n.a. & TTCGATCAAGGGGAG \\
\hline
\end{tabular}

${ }^{a}$ Size for left/right LTR.

${ }^{b}$ Shading indicates direct repeat formed on integration.

${ }^{c}$ h.a., data not available.

from rice. ${ }^{30,45,46}$ A new RT sequence alignment was prepared, including a representative of each of the 39 families as well as the other rice $r t$ sequences that are available in Genbank. We also included, in this alignment, some RT sequences from known elements of species other than $O$. sativa. The alignment was used to produce a phylogenetic tree, shown in Figure 2. Many of the families discovered by our analysis of the $O$. sativa sequence are novel, having no representatives in Genbank. Of these, families 9A and 9B are fully distinct from all other RT clades. Only one deeply branching clade in the tree shown in Figure 2, the one including Tst1 (potato), PDR1 (pea), and Tos10 (rice), has both a rice member and no family member from our analysis. In total, five to 10 additional families are present among the cloned PCR fragments in the database but not in the genomic sequences we have scanned. This suggests that the total number of families in rice genome is probably more than 50 .

Conversely, all of the previously characterized Oryza retrotransposons could be assigned to one of the families we found: RIRE1 belongs to family $6 \mathrm{C}$, Tos 17 to $3 \mathrm{~A}$, and Retrofit probably to a new family in class 2 . These results were verified by comparisons at the DNA level. Some of the PCR fragments can also be assigned to some of the families, or at least to classes. For example, Tos6 can be placed in $6 \mathrm{~A}$, Tos 15 in $7 \mathrm{~A}$, and Tos 14 in $7 \mathrm{~B}$ or $7 \mathrm{C}$. Some of the RT classes are not represented by the cloned PCR fragments in the database (classes 1, 5, and 9). This could reflect a sampling problem in the PCR methods used to produce the $r t$ sequences, but the classes not represented in the database are not necessarily the least abundant. That suggests that the PCR protocols used for copia-like rts cannot amplify certain classes of $r t$, producing an underestimation of the sequence variability of copia-like elements.

Regarding retrotransposons from other plants, similarities with rice sequences were not high but were nevertheless sufficient to associate the elements with one or more rice classes. Opie2 (maize), Endovir1 (Arabidopsis thaliana), and SIRE-1 (soybean) are related to class 1. SIRE-1 and Endovir1 are retrovirus-like retrotransposons, meaning that they contain a region upstream of the pol gene that is similar to an env gene. The presence of an envelope domain expands the internal domain, and, interestingly, it is class 1 elements in rice that are the ones with longer internal domains. Hopscotch (maize) is associated with class 2, Stonor (maize) with class 6 , and BARE-1 (barley) with 6C. Finally, the retrotransposons Tto1 (tobacco), Tnt1 (tobacco), and Ta1 (A. thaliana) are related to class 8 .

\subsection{Expression of Copia-Like Retrotransposons in Rice}

To search globally for expression of the retroelement families identified by their $r t$ sequences, all of the elements 


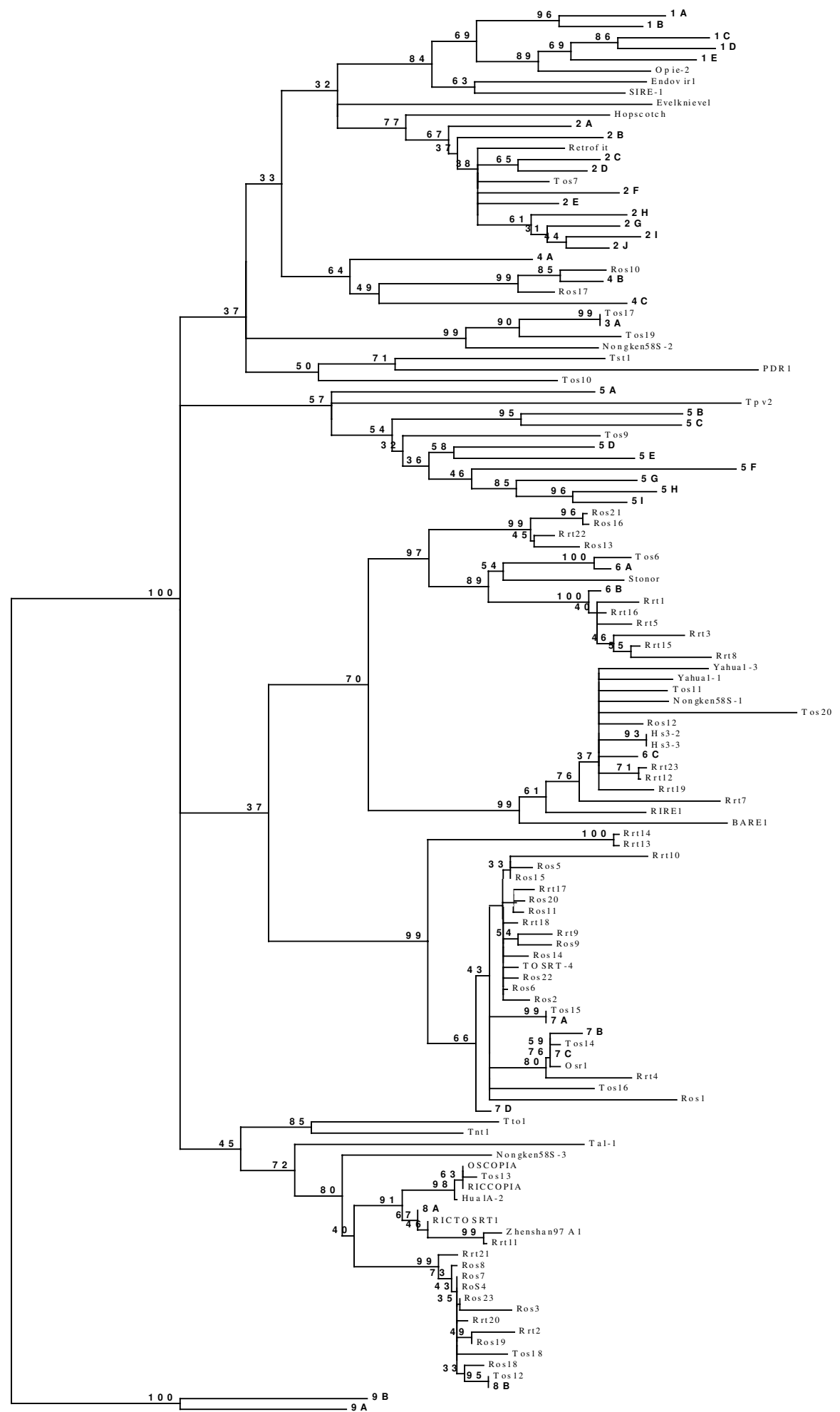

Figure 2. Neighbor-joining tree of the rice and other plant copia-like reverse transcriptase amino acid sequences. Bootstraps were performed with 1000 repetitions; branches with bootstrap values under 30\% are shown as unresolved. Representatives of the rice families from Figure 1 are shown in bold. The PCR clones from the database are Ros, ${ }^{45} \mathrm{Rrt},{ }^{46}$ Tos, ${ }^{30}$ RICTOSRT1 (accession D12825), RICCOPIA (M94492), OSCOPIA (M94492), TOSRT4 (D12826.1), Hua1A-2 (AF349737), Hs3-2 (AF349740), Hs3-3 (AF349741), Nongken58s-1 (AF349742), Nongken58s-2 (AF349743), Nongken58s-3 (AF349744), Yahua1-1 (AF349745), Yahua1-3 (AF349747), Zhenshan97A-1 (AF349748), Zhenshan97A-3 (AF349750), and Zhenshan97A-2 (AF349749). The characterized retrotransposons are Tst1 (X52287), PDR1 (AJ243356), Opie2 (U68408), Endovir1 (AY016208), SIRE-1 (AF053008), Evelknievel (AF039376), Hopscotch (U12626), Retrofit (U72726), Tos17 (D88394), Tpv2 (AJ005762), Stonor (AF082133), RIRE1 (D85598), BARE-1 (Z17327), Osr1 (AB046118), Tto1 (D12827), Ta1 (X13291), and Tnt1 (X13777). 
Table 2. Rice copia-Like retrotransposon families and their matching grass ESTs.

\begin{tabular}{|c|c|c|c|c|c|c|c|c|c|c|c|c|}
\hline \multirow[b]{3}{*}{ Class } & \multirow[b]{3}{*}{ Family } & \multicolumn{11}{|c|}{ ESTs } \\
\hline & & \multicolumn{6}{|c|}{ Oryza $^{a}$} & \multirow[b]{2}{*}{ Sorghum } & \multirow[b]{2}{*}{ Zea } & \multirow[b]{2}{*}{ Triticum } & \multirow[b]{2}{*}{ Hordeum } & \multirow[b]{2}{*}{ Secale } \\
\hline & & Total & $\mathrm{V}$ & $\mathrm{F}$ & $\mathrm{G}$ & $\mathrm{S}$ & $\mathrm{T}$ & & & & & \\
\hline \multirow[t]{5}{*}{1} & A & 5 & 1 & - & 1 & - & 3 & - & - & - & 2 & - \\
\hline & B & 2 & - & - & 1 & - & 1 & - & - & - & - & - \\
\hline & $\mathrm{C}$ & - & - & - & - & - & - & - & 1 & - & - & - \\
\hline & $\mathrm{D}$ & 1 & 1 & - & - & - & - & 1 & 1 & - & - & - \\
\hline & $\mathrm{E}$ & - & - & - & - & - & - & - & 5 & - & - & - \\
\hline \multirow[t]{10}{*}{2} & A & 5 & 3 & 1 & - & - & 1 & - & - & - & - & - \\
\hline & B & - & - & - & - & - & - & - & - & - & - & - \\
\hline & $\mathrm{C}$ & - & - & - & - & - & - & - & - & - & - & - \\
\hline & $\mathrm{D}$ & - & - & - & - & - & - & - & - & - & - & - \\
\hline & $\mathrm{E}$ & 2 & 2 & - & - & - & - & - & - & 2 & 1 & - \\
\hline & $\mathrm{F}$ & - & - & - & - & - & - & - & - & - & 1 & - \\
\hline & $\mathrm{G}$ & - & - & - & - & - & - & - & - & - & - & - \\
\hline & $\mathrm{H}$ & - & - & - & - & - & - & - & - & - & - & - \\
\hline & I & 3 & 1 & 1 & - & 1 & - & - & 1 & - & - & - \\
\hline & $\mathbf{J}$ & - & - & - & - & - & - & - & - & - & 1 & - \\
\hline 3 & A & 2 & - & - & - & - & 2 & - & - & 1 & - & - \\
\hline \multirow[t]{3}{*}{4} & A & - & - & - & - & - & - & 2 & - & - & - & - \\
\hline & B & 3 & 1 & - & 1 & - & 1 & - & - & - & 3 & - \\
\hline & $\mathrm{C}$ & - & - & - & - & - & - & - & 1 & - & - & - \\
\hline \multirow[t]{9}{*}{5} & A & - & - & - & - & - & - & - & - & - & - & - \\
\hline & B & 2 & - & 2 & - & - & - & - & - & - & - & - \\
\hline & $\mathrm{C}$ & 1 & - & 1 & - & - & - & - & - & - & - & - \\
\hline & $\mathrm{D}$ & 1 & - & - & - & 1 & - & - & - & - & - & - \\
\hline & $\mathrm{E}$ & - & - & - & - & - & - & 1 & - & - & - & - \\
\hline & $\mathrm{F}$ & 1 & - & 1 & - & - & - & - & - & - & - & - \\
\hline & $\mathrm{G}$ & - & - & - & - & - & - & - & - & - & - & - \\
\hline & $\mathrm{H}$ & 2 & - & 2 & - & - & - & 6 & 1 & - & - & - \\
\hline & $\mathrm{I}$ & 5 & 5 & - & - & - & - & 3 & 1 & 1 & 3 & - \\
\hline \multirow[t]{3}{*}{6} & I & - & - & - & - & - & - & - & - & - & - & - \\
\hline & B & 9 & 1 & 4 & 1 & - & 3 & 1 & - & 1 & - & - \\
\hline & $\mathrm{C}$ & 9 & - & 2 & 2 & - & 5 & - & - & - & 7 & - \\
\hline \multirow[t]{4}{*}{7} & A & - & - & - & - & - & - & - & - & - & - & - \\
\hline & B & 6 & - & 2 & - & 3 & 1 & 1 & 1 & 1 & - & - \\
\hline & $\mathrm{C}$ & 2 & - & 1 & - & - & 1 & 1 & - & - & - & - \\
\hline & $\mathrm{D}$ & - & - & - & - & - & - & - & 1 & - & 4 & 1 \\
\hline \multirow[t]{2}{*}{8} & A & 1 & 1 & - & - & - & - & 1 & - & - & - & 1 \\
\hline & B & 2 & 1 & 1 & - & - & - & - & - & - & - & - \\
\hline \multirow[t]{2}{*}{9} & A & - & - & - & - & - & - & - & - & - & - & - \\
\hline & B & 1 & - & - & 1 & - & - & - & - & - & 1 & - \\
\hline
\end{tabular}

${ }^{a} \mathrm{~V}$, vegetative tissues; F, floral tissues; G, grain and related tissues; $\mathrm{S}$, stress-induced tissues; $\mathrm{T}$, tissue culture.

identified were used as query sequences against the EST database. The matches were analyzed and are displayed in Table 2. Only ESTs from grasses were considered. A total of 65 rice ESTs were identified with a high probability of match, in addition to 23 from barley, 17 from sorghum, 13 from maize, six from wheat, and two from rye. The number of rice ESTs similar to each of the families was not correlated with their genomic copy numbers. For example, $6 \mathrm{~B}$ and $6 \mathrm{C}$ are the most represented in the EST databases, although we detected only three copies of each, whereas the most abundant in the genomic sequences, family $7 \mathrm{D}$, does not have any rice-related EST. The 2A family, which has only one element in the genomic sequences, has six related ESTs.

These results suggest that possible genomic contamination in the EST databases has not been sufficient to make the distribution of elements in them mirror that in the genome. Rather, the EST frequencies are indicative of transcriptional activity of the families of copialike retrotransposons in rice. These results beg the question of why highly prevalent elements, whose abundance ultimately results from expression, should be rare among 
the ESTs. This discordance may be explained in part by the fact that the tissues used for mRNA isolation were not the ones in which the abundant families were most highly expressed. Conversely, it is well known that rare retrotransposons such as Tos17 can be highly expressed under particular conditions. ${ }^{30}$ Only two ESTs similar to Tos17 (family 3A) were found; both are derived from tissue culture cDNA libraries. Furthermore, the finding of ESTs from other grasses matching rice retroelement RT classes or families indicates that some of these are both present and expressed in all grasses and is consistent with earlier, broader analyses. ${ }^{10}$

\subsection{Identification and Frequency of Other Classes of Retrotransposons in the Rice Genome}

Gypsy-like elements are the other major class of LTR retrotransposons, and some perspective on their occurrence in the rice genome is useful for comparison with the copialike elements. Using a method similar to that for copia-like elements, we were able to estimate the number of gypsylike and LINE-like retroelements based on their $r t$ domains. We found a total of 222 gypsy-like elements, in the fraction of the genome surveyed, indicating about 2220 gypsy-like elements in the genome as a whole. This is twice the number of copia-like ones, compared with previous estimates of a three- to fivefold greater abundance. ${ }^{28,47}$ Two thousand copies, at an average size of $7.7 \mathrm{~kb}$, comprise about $3.6 \%$ of the genome, although this may be an underestimation because we did not consider solo LTRs and truncated elements. The copia-like retrotransposons and their deletion derivatives, together with the gypsy-like elements, amount to more than 4800 copies or about $5.9 \%$ of the genome, at an average spacing of one element every $84 \mathrm{~kb}$.

Our search also produced 66 LINE-like (non-LTR) retrotransposons, indicating about 660 LINEs in the genome, and five integrated pararetroviruses. LINE elements have previously been reported as features of the Oryza genome. ${ }^{39,48}$ Pararetroviruses, lacking their own integrase, have been found in the genomes of both banana ${ }^{49}$ and tobacco. $^{50}$ Our identification of a few sequences in the rice genome shows that the presence of pararetrovirus sequences in the genome is not unique to tobacco and banana.

\section{CONCLUSIONS}

That genome sequencing projects are qualitatively changing our understanding of the mechanisms of evolution of retrotransposons has been demonstrated for Saccharomyces cerevisiae, ${ }^{51,52}$ Caenorhabditis elegans, ${ }^{53,54}$ and A. thaliana. ${ }^{55,56}$ The increasing genomic sequence information for rice now provides an excellent opportunity to understand the evolution and diversity of transposable elements in grasses. Grasses are interesting subjects because of the wide range in the size of their genomes and the prevalence of their retrotransposons. In this work, we concentrated on the analysis of copia-class elements, which includes Ty1 of yeast.

Our analysis is based on about $10 \%$ of the rice genome. A limitation of the data, true also for the "finished" A. thaliana genome, is that centromeres, which tend to be rich in repetitive DNA (in some cases including transposable elements), ${ }^{33,57-60}$ are relatively underrepresented in the database. While the final word on the rice genome awaits complete annotation of the entire genome, including centromeres, the available data may be sufficient to give a good general view of the genome, excluding the centromeres and telomeres.

Our analysis of the data yields three main conclusions. First, although the number of retrotransposons in rice is considerable, it is still very low compared with that in other members of the grass family and in other plants generally. The contribution of retrotransposons to the genome share in rice is similar to that in A. thaliana. Although copy number is relatively low, variability is not, with at least 39 different families grouped into at least nine classes. A high degree of heterogeneity in copia-like retrotransposons appears, from other available data, $5,6,8,30,35,36,45,61-63$ to be typical for plants, including $A$. thaliana. Finally, our and others' data ${ }^{56}$ indicate that related classes of copia-like retrotransposons can be found in several plant species, within the genomes of both monocots and dicots. This indicates that copia-like elements already comprised distinct families of elements before the divergence of the monocots and dicots, placing their origin at more than 200 million years ago, and that some of these ancient families have persisted until the present day.

Despite the persistence of diverse families of copialike elements in the rice genome, their copy number is exceptionally low compared with that in other cereals. For example, RIRE1 belongs to the same family (6C) as $B A R E-1$ and Wis2 from barley and wheat. ${ }^{31,64}$ This family is highly abundant in both species ${ }^{20,65}$ but is represented by only about 30 copies in rice (about 90 if we consider all class 6 elements together). In the wild rice Oryza australiensis, with a genome size double that of rice, the number of RIRE1 copies is in the thousands. ${ }^{29}$ The variation in the number of RIRE1 copies alone can explain about one-third of the difference in size between the genomes of $O$. sativa and $O$. australiensis. A comparison of a contiguous region of the barley and rice genomes ${ }^{66}$ indicates that the larger genome of barley can be attributed at least in part to the integration of retrotransposons into currently retrotransposon-poor regions of the rice genome. The key issue emerging from these comparisons is whether the genome of cultivated rice never expanded through gain of retrotransposon copies, or whether, instead, it expanded but then lost the copies integrated earlier. 
The steady-state copy number of a retrotransposon depends on the balance between the rates of gain through retrotransposition and loss through mutation and through various forms of deletion. Retrotransposition requires transcription, reverse transcription, and, ultimately, integration. In general, retrotransposons in rice seem to be poorly transcribed compared with elements in other grasses and, by the lack of copy number variation between cultivars, ${ }^{67}$ fairly quiescent integrationally. Only $0.98 \%$ ( 0.98 per thousand) of all of the rice ESTs in the databases matched retrotransposons, compared with the $3.12 \%$ o for barley, $2.49 \%$ for maize, and $1.26 \%$ for wheat. ${ }^{10}$ All rice retrotransposons examined by Hirochika and co-workers ${ }^{30}$ were inactive under normal growth conditions, and only three were activated by tissue culture. One of these, Tos 17 (family 3C) is rare, despite its potential activity. ${ }^{30}$ Family 6C (RIRE1) is the second most common among the ESTs after 6B, suggesting that it, at least, should be transcriptionally active compared with other families, but the family's genomic prevalence is not commensurately great.

Not only may rice retrotransposons not be particularly actively transcribed; they also may be lost through ectopic or unequal recombination. Intrachromosomal recombination between retrotransposon LTRs can remove the internal domain of an element, producing a solo LTR. Recombination between LTRs not of the same copy can remove, as well, intervening DNA and generate a solo LTR. ${ }^{4,21}$ This process appears to be common for retrotransposons in barley $^{20,68}$ and to play a role in modulating copy number in populations of wild barley, $H$. spontaneum. ${ }^{16}$ The maize genome, however, appears to contain relatively few solo LTRs. ${ }^{18}$

We are able to derive some conclusions from our data regarding recombinational loss of retrotransposons in rice. For the RIRE1 element (family 6C), we found three elements containing RT and LTRs, 25 solo LTRs, and one element with LTRs but a deletion in the RT domain. This amounts to a total of 33 LTRs and four internal regions, or 330 RIRE1 LTRs in the genome as a whole, comparable to the previous estimate of 180 RIRE1 LTRs. ${ }^{29}$ Hence, the rice genome has an LTR:internal domain ratio for RIRE1 of $8.25: 1$ instead of the $2: 1$ expected if there were no solo LTRs. Considering all copia-like retroelements in the genome, our data yield an LTR:internal domain ratio of 3.6:1 and suggest that more copia-like retrotransposons than are currently in the genome may have been lost by recombination. Furthermore, although for the gypsy-like RIRE2 we find relatively few solo LTRs and a LTR:internal domain ratio of 2.3 , the centromere of chromosome 5 in rice contains many repeats similar to solo LTRs of gypsy-like RIRE3, RIRE7, and RIRE8. ${ }^{59}$ These calculations and data together indicate that intrachromosomal, ectopic recombination between LTRs is driving much of the loss of retroelements from the rice genome.
If recombinational loss of retrotransposons is a factor shaping the rice genome, it may explain the high degree of heterogeneity we find among copia-like elements. It is thought that retrotransposon heterogeneity in plants is a consequence of their prevalence. ${ }^{69}$ For rice as well as for A. thaliana, ${ }^{35}$ the currently high diversity but low copy number therefore may be a remnant of an earlier, larger genome and population of retrotransposons. The long-term evolutionary dynamics of mobile elements remain a matter of debate, ${ }^{4,70}$ and the factors controlling gain and loss of retrotransposons need to be clarified. Detailed analysis of whole genomes, such as that of $O$. sativa, in comparison with genomic regions of related species, ${ }^{66}$ is expected to shed some light on genome dynamics and the processes underlying it.

Acknowledgments: This work was carried out with support from the Academy of Finland under projects 44404 and 47581 .

\section{References and Notes}

1. A. Kumar and J. Bennetzen, Plant retrotransposons, Annu. Rev. Genet. 33, 479 (1999).

2. Y. Xiong and T. H. Eickbush, Origin and evolution of retroelements based upon their reverse transcriptase sequences, ЕMBO J. 9, 3353 (1990).

3. T. Schmidt, LINEs, SINEs and repetitive DNA: non-LTR retrotransposons in plant genomes, Plant Mol. Biol. 40, 903 (1999).

4. J. Bennetzen, Transposable element contributions to plant gene and genome evolution, Plant Mol. Biol. 42, 251 (2000).

5. A. J. Flavell, D. B. Smith, and A. Kumar, Extreme heterogeneity of Ty1-copia group retrotransposons in plants, Mol. Gen. Genet. 231, 233 (1992)

6. H. Hirochika and R. Hirochika, Ty1-copia group retrotransposons as ubiquitous components of plant genomes, Jpn. J. Genet. 68, 35 (1993).

7. A. Suoniemi, J. Tanskanen, and A. H. Schulman, Gypsy-like retrotransposons are widespread in the plant kingdom, Plant J. 13, 699 (1998).

8. D. F. Voytas, M. P. Cummings, A. K. Konieczny, F. M. Ausubel, and S. R. Rodermel, Copia-like retrotransposons are ubiquitous among plants, Proc. Natl. Acad. Sci. USA 89, 7124 (1992).

9. A. Suoniemi, A. Narvanto, and A. H. Schulman, The BARE-1 retrotransposon is transcribed in barley from an LTR promoter active in transient assays, Plant Mol. Biol. 31, 295 (1996).

10. C. M. Vicient, M. Jääskeläinen, R. Kalendar, and A. H. Schulman, Active retrotransposons are a common feature of grass genomes, Plant Physiol. 125, 1283 (2001).

11. T. Beguiristain, M. A. Grandbastien, P. Puigdomènech, and J. M Casacuberta, Three Tnt1 subfamilies show different stress-associated patterns of expression in tobacco. Consequences for retrotransposon control and evolution in plants, Plant Physiol. 127, 212 (2001)

12. S. R. Wessler, Turned on by stress. Plant retrotransposons, Curr. Biol. 6, 959 (1996).

13. M.-A. Grandbastien, Activation of retrotransposons under stress conditions, Trends Plant Sci. 3, 181 (1998).

14. H. Okamoto and H. Hirochika, Efficient insertion mutagenesis of Arabidopsis by tissue culture-induced activation of the tobacco retrotransposon Tto1, Plant J. 23, 291 (2000).

15. M. A. Grandbastien, C. Audeon, J. M. Casacuberta, P. Grappin, H. Lucas, C. Moreau, and S. Pouteau, Functional analysis of the tobacco Tnt1 retrotransposon, Genetica 93, 181 (1994) 
16. R. Kalendar, J. Tanskanen, S. Immonen, E. Nevo, and A. H. Schulman, Genome evolution of wild barley (Hordeum spontaneum) by BARE-1 retrotransposon dynamics in response to sharp microclimatic divergence, Proc. Natl. Acad. Sci. USA 97, 6603 (2000).

17. M. Jääskeläinen, A.-H. Mykkänen, T. Arna, C. Vicient, A. Suoniemi, R. Kalendar, H. Savilahti, and A. H. Schulman, Retrotransposon BARE-1: expression of encoded proteins and formation of virus-like particles in barley cells, Plant J. 20, 413 (1999).

18. P. SanMiguel, B. S. Gaut, A. Tikhonov, Y. Nakajima, and J. L. Bennetzen, The paleontology of intergene retrotransposons in maize, Nat. Genet. 20, 43 (1998).

19. M. D. Bennett and I. J. Leitch, Plant DNA C-values Database (http://www.rbgkew.org.uk/cval/homepag e.html) (2001).

20. C. M. Vicient, A. Suoniemi, K. Anamthawat-Jónsson, J. Tanskanen, A. Beharav, E. Nevo, and A. H. Schulman, Retrotransposon BARE-1 and its role in genome evolution in the genus, Hordeum, Plant Cell 11, 1769 (1999).

21. D. Petrov, Slow but steady: reduction of genome size through biased mutation, Plant Cell 9, 1900 (1997).

22. K. Arumuganathan and E. D. Earle, Nuclear DNA content of some important plant species, Plant Mol. Biol. Rep. 9, 208 (1991).

23. A. Barakat, N. Carels, and G. Bernardi, The distribution of genes in the genomes of Gramineae, Proc. Natl. Acad. Sci. USA 94, 6857 (1997).

24. A. P. Tikhonov, P. J. SanMiguel, Y. Nakajima, N. M. Gorenstein, J. L. Bennetzen, and Z. Avramova, Colinearity and its exceptions in orthologous adh regions of maize and sorghum, Proc. Natl. Acad. Sci. USA 96, 7409 (1999).

25. V. G. Deshpande and P. K. Ranjekar, Repetitive DNA in three Gramineae species with low DNA content, Hoppe Seylers Z. Physiol. Chem. 361, 1223 (1980).

26. H. Hirochika, A. Fukuchi, and F. Kikuchi, Retrotransposon families in rice, Mol. Gen. Genet. 233, 209 (1992).

27. S. Wang, N. Liu, K. Peng, and Q. Zhang, The distribution and copy number of copia-like retrotransposons in rice (Oryza sativa L.) and their implications in the organization and evolution of the rice genome, Proc. Natl. Acad. Sci. USA 96, 6824 (1999).

28. K. Turcotte, S. Srinivasan, and T. Bureau, Survey of transposable elements from rice genomic sequences, Plant J. 25, 169 (2001).

29. R. Nakajima, K. Noma, H. Ohtsubo, and E. Ohtsubo, Identification and characterization of two tandem repeat sequences (TrsB and $\operatorname{Trs} C)$ and a retrotransposon (RIRE1) as genome-general sequences in rice, Genes Genet. Syst. 71, 373 (1996).

30. H. Hirochika, K. Sugimoto, Y. Otsuki, H. Tsugawa, and M. Kanda, Retrotransposons of rice involved in mutations induced by tissue culture, Proc. Natl. Acad. Sci. USA 93, 7783 (1996).

31. K. Noma, R. Nakajima, H. Ohtsubo, and E. Ohtsubo, RIRE1, a retrotransposon from wild rice Oryza australiensis, Genes Genet. Syst. 72, 131 (1997).

32. H. Ohtsubo, N. Kumekawa, and E. Ohtsubo, RIRE2, a novel gypsylike retrotransposon from rice, Genes Genet. Syst. 74, 83 (1999).

33. N. Kumekawa, H. Ohtsubo, T. Horiuchi, and E. Ohtsubo, Identification and characterization of novel retrotransposons of the gypsy type in rice, Mol. Gen. Genet. 260, 593 (1999).

34. N. Kumekawa, N. Ohmido, K. Fukui, E. Ohtsubo, and H. Ohtsubo, A new gypsy-type retrotransposon, RIRE7: preferential insertion into the tandem repeat sequence TrsD in pericentromeric heterochromatin regions of rice chromosomes, Mol. Genet. Genomics 265, 480 (2001).

35. A. Konieczny, D. F. Voytas, M. P. Cummings, and F. M. Ausubel, A superfamily of Arabidopsis thaliana retrotransposons, Genetics 127, 801 (1991).

36. P. L. VanderWiel, D. F. Voytas, and J. F. Wendel, Copia-like retrotransposable element evolution in diploid and polyploid cotton (Gossypium L.), J. Mol. Evol. 36, 429 (1993).
37. M. Yañez, I. Verdugo, M. Rodriguez, S. Prat, and S. Ruiz-Lara, Highly heterogeneous families of Ty1/copia retrotransposons in the Lycopersicum chilense genome, Gene 222, 223 (1998).

38. S. F. Altschul, W. Gish, W. Miller, E. W. Myers, and D. J. Lipman, Basic local alignment search tool, J. Mol. Biol. 215, 403 (1990).

39. S. E. Kubis, J. S. Heslop-Harrison, C. Desel, and T. Schmidt, The genomic organization of non-LTR retrotransposons (LINEs) from three Beta species and five other angiosperms, Plant Mol. Biol. 36, 821 (1998).

40. J. D. Thompson, D. G. Higgins, and T. J. Gibson, Clustal W: improving the sensitivity of progressive multiple sequence alignment through sequence weighting, position specific gap penalties and weight matrix choice, Nucleic Acids Res. 22, 4673 (1994).

41. Y. Van de Peer and R. De Wachter, TREECON for Windows: a software package for the construction and drawing of evolutionary trees for the Microsoft Windows environment, Comput. Appl. Biosci. 10, 569 (1994).

42. N. Saitou and M. Nei, The neighbor-joining method: a new method for reconstructing phylogenetic trees, Mol. Biol. Evol. 4, 406 (1987).

43. D. H. Lankenau, P. Huijser, E. Jansen, K. Miedema, and W. Hennig, DNA sequence comparison of micropia transposable elements from Drosophila hydei and Drosophila melanogaster, Chromosoma 99, 111 (1990).

44. N. V. Lyubomirskaya, I. R. Arkhipova, Y. V. Ilyin, and A. I. Kim, Molecular analysis of the gypsy (mdg4) retrotransposon in two Drosophila melanogaster strains differing by genetic instability, Mol. Gen. Genet. 223, 305 (1990).

45. Y. Matsuoka and K. Tsunewaki, Evolutionary dynamics of the Ty1copia group retrotransposons in grass shown by reverse transcriptase domain analysis, Mol. Biol. Evol. 16, 208 (1999).

46. S. Wang, Q. Zhang, P. J. Maughan, and M. A. Saghai Maroof, Copialike retrotransposons in rice: sequence heterogeneity, species distribution and chromosomal locations, Plant Mol. Biol. 33, 1051 (1997).

47. L. Mao, T. C. Wood, Y. Yu, M. A. Budiman, J. Tomkins, S. Woo, M. Sasinowski, G. Presting, D. Frisch, S. Goff, R. A. Dean, and R. A. Wing, Rice transposable elements: a survey of 73,000 sequencetagged connectors, Genome Res. 10, 982 (2000).

48. K. Noma, E. Ohtsubo, and H. Ohtsubo, Non-LTR retrotransposons (LINEs) as ubiquitous components of plant genomes, Mol. Gen. Genet. 261, 71 (1999).

49. G. Harper, J. O. Osuji, J. S. Heslop-Harrison, and R. Hull, Integration of banana streak badnavirus into the Musa genome: molecular and cytogenetic evidence, Virology 255, 207 (1999).

50. J. Jakowitsch, M. F. Mette, J. van Der Winden, M. A. Matzke, and Matzke, A. J. Matzke, Integrated pararetroviral sequences define a unique class of dispersed repetitive DNA in plants, Proc. Natl. Acad. Sci. USA 96, 13241 (1999).

51. J. M. Kim, S. Vanguri, J. D. Boeke, A. Gabriel, and D. F. Voytas, Transposable elements and genome organization: a comprehensive survey of retrotransposons revealed by the complete Saccharomyces cerevisiae genome sequence, Genome Res. 8, 464 (1998).

52. I. K. Jordan and J. F. McDonald, Comparative genomics and evolutionary dynamics of Saccharomyces cerevisiae Ty elements, Genetica 107, 3 (1999).

53. T. Oosumi, B. Garlick, and W. R. Belknap, Identification of putative nonautonomous transposable elements associated with several transposon families in Caenorhabditis elegans, J. Mol. Evol. 43, 11 (1998).

54. N. J. Bowen and J. F. McDonald, Genomic analysis of Caenorhabditis elegans reveals ancient families of retroviral-like elements, Genome Res. 9, 924 (1999).

55. Q. H. Le, S. Wright, Z. Yu, and T. Bureau, Transposon diversity in Arabidopsis thaliana, Proc. Natl. Acad. Sci. USA 97, 7376 (2000).

56. I. Marín and C. Lloréns, Ty3/Gypsy retrotransposons: description of new Arabidopsis thaliana elements and evolutionary perspectives derived from comparative genomic data, Mol. Biol. Evol. 17, 1040 (2000). 
57. M. G. Francki, Identification of Bilby, a diverged centromeric Ty1copia retrotransposon family from cereal rye (Secale cereale L.), Genome 44, 266 (2001).

58. N. Kumekawa, T. Hosouchi, H. Tsuruoka, and H. Kotani, The size and sequence organization of the centromeric region of Arabidopsis thaliana chromosome 5, DNA Res. 7, 315 (2000).

59. K. Nomomura and N. Kurata, The centromere composition of multiple repetitive sequences on rice chromosome 5, Chromosoma 110, 284 (2001).

60. G. G. Presting, L. Malysheva, J. Fuchs, and I. Schubert, A Ty3/gypsy retrotransposon-like sequence localizes to the centromeric regions of cereal chromosomes, Plant J. 16, 721 (1998).

61. Y. Matsuoka and K. Tsunewaki, Wheat retrotransposon families identified by reverse transcriptase domain analysis, Mol. Biol. Evol. 13, 1384 (1996).

62. Y. Matsuoka and K. Tsunewaki, Presence of wheat retrotransposons in Gramineae species and the origin of wheat retrotransposon families, Genes Genet. Syst. 72, 335 (1997).

63. V. Kapitonov and J. Jurka, Molecular paleontology of transposable elements from Arabidopsis thaliana, Genetica 107, 27 (1999).
64. C. M. Vicient, R. Kalendar, K. Anamthawat-Jonsson, and A. H. Schulman, Structure, functionality, and evolution of the BARE-1 retrotransposon of barley, Genetica 107, 53 (1999).

65. G. Moore, H. Lucas, N. Batty, and R. Flavell, A family of retrotransposons and associated genomic variation in wheat, Genomics 10, 461 (1991).

66. J. Dubcovsky, W. Ramakrishna, P. J. SanMiguel, C. S. Busso, L. Yan, B. A. Shiloff, and J. L. Bennetzen, Comparative sequence analysis of colinear barley and rice bacterial artificial chromosomes, Plant Physiol. 125, 1342 (2001).

67. H. Hirochika, Retrotransposons of rice: their regulation and use for genome analysis, Plant Mol. Biol. 35, 231 (1997).

68. K. Shirasu, A. H. Schulman, T. Lahaye, and P. Schulze-Lefert, A contiguous $66 \mathrm{~kb}$ barley DNA sequence provides evidence for reversible genome expansion, Genome Res. 10, 908 (2000).

69. B. Charlesworth, Genetic divergence between transposable elements, Genet. Res. 48, 111 (1986).

70. W. J. Miller, J. F. McDonald, and W. Pinsker, Molecular domestication of mobile elements, Genetica 100, 261 (1997). 